\title{
GEOGRAPHICAL DISTRIBUTION OF MEMBERS
}

\author{
Auburn \\ Wilson, R. L. \\ Birmingham \\ Hess \\ Miles, E. P. \\ Rayl \\ Fort McClellan \\ Rosenbaum, I. \\ Linden \\ Adams, G. J.
}

\section{Flagstaff \\ Lampland \\ Mehlenbacher}

Caraher

\section{Conway}

Altadena
Dix Atascadero
Schmiedel
Berkeley
Robinson, J. B.
Univ. of California
Barankin
Benson
Bernstein, B. A.
Bernstein, D. L.
Buck
Dresch
Evans, G. C.
Foster, A. L.
Frankel, S. P.
Goldsworthy
Hallam
Horn
Irwin, F.
Lehmer
Lenzen
Leuschner
Levy, S. H.
Lewis, J. V.
Lewy
Mann, W. R.
Morrey
Morse, A. P.
Mowbray.
Nelson, M. L.
Neyman
Noble, C. A.
Putnam, T. M.
Robinson, R. M.
Schaaf

ALABAMA

Marion
Glass
Maxwell Field
Miller, R. A.
Mobile
Gerhardt
Montevallo
Jackson, R. L.

Watson

Gonzalez

Brothers

Univ. of Alabama

Cassity

\section{ARIZONA}

\begin{tabular}{|c|c|}
\hline Phoenix & Mickelson \\
\hline Williams, J. D. & $\begin{array}{l}\text { Univ. of } A r \\
\text { Boldyreff }\end{array}$ \\
\hline
\end{tabular}

\section{ARKANSAS}

$\begin{array}{cl}\text { Fayetteville } & \text { Comfort } \\ \text { Univ. of Arkansas } & \text { Hosford } \\ \text { Adkisson } & \text { Kent }\end{array}$

\section{CALIFORNIA}

Shephard

Simons, W. H

Singer, P. M.

Sperry

Swinford

Tarski

Williams, A. R.

Wolf, F.

Wong, B. C.

Perlis Burbank

Zilmer

$$
\text { Camp Haan }
$$

Arnold, J. W. Chula Vista

Langhaar Claremont

Hamilton, H. J. Jaeger, C. G.

$$
\text { Davis }
$$

Univ. of California

Baker, G. A.

Mersman

Roessler El Segundo

Forsythe, A. I. Fuller Emeryville

Fresno

Morris, F. R.

Wakerling

$$
\text { Glendale }
$$

Rahn

White, $\mathrm{P}$. Los Angeles

Harrington, C. $\mathrm{H}$

Roberts, W. C. Grass Valley

Hofmann
Montgomery

Spring Hill

Talladega

University

Hayward

Inglewood

$$
\text { La Jolla }
$$

McEwen, G. F.

Ablow

Elder, C. C.

Humm

Quilliam

White, R. L.

George Pepperdine

Coll.

Hinds

Immaculate Heart Coll.

Collier

Green, A. A.

Los Angeles City Coll.

Brady, C. P.

Duncan

Urner

Occidental Coll.

Dieckmann

Wheeler, D. B.

Univ. of California

at Los Angeles

Arthur

Be11, C.

Charney

Hummel

Kennedy, E. S.

Lewis, F. A.

Mancill

Ott, W. P.

Thurston

Wade, T. L.

Warnock

Hohn

Leonard

Mewborn

Purcell

Nichols

Richardson, D. P

Hoel

Hunt, G. H.

James, G.

Lass

Mason, W. E.

Puckett

Sherwood

Simmons, D. H.

Taylor, A. E.

Taylor, B. P.

Thomas, T. Y.

Valentine

Whyburn, W. M.

Worthington

Zorn

Univ. of Southern California

Ames, L. D.

Butter

Nelson, J. B.

Steed

Marston

\section{Monterey}

\section{Pasadena}

White, M. B.

Woolson

California Inst. of Tech.

Bateman, $\mathrm{H}$.

Bell, E. T.

Birchby

Harrison, G.

Houston

Hyers

James, R. C.

Daus

Glazier

Hedrick 


Mason, M.
Michal
Mills
Millsaps
Panofsky
Shand
Snowdon
Tolman
Van Buskirk
Ward, M.
Wear
Pasadena Junior
Glenn Coll.
$\quad$ Redlands
Albert, O. W.

Albert, O. W.

\section{COLORADO}

$\quad$ Boulder
Univ. of Colorado
Britton
DeLong
Hutchinson, C. A.
Kempner
Kendall

\section{Bridgeport}

Escott, F. H.

Turner, M. H.

Ashman

$$
\text { Darien }
$$

East Hartford

Isaacs

\section{Hartford}

Doyle, M. E.

E1ston

Keffer

Larus

Satterthwaite

White, M. E. Trinity Coll.

Dadourian

Downs

Mitchell, A. K.

Wyckoff Lakeville

Northrop

Newark
Univ. of Delaware

Cannon

Washington
Alsberg
Blake, A.
Bush, V.
Claytor
Cohen, L.

$\quad$ San Diego
Burke, B. H.
Klauber
McConnell, H. J.
San Diego State
$\quad$ Coll.
Walton, L. F.

Walton, L. F.

\section{San Francisco}

Brennan

Dean, J. P.

Hayes, C. A.

Westbrook

San Francisco

Junior Coll.

McCarty

Noble, A. R. Univ. of San Francisco

Saunders

Heaslet San Jose

Myers, W. H. Santa Barbara Rauch, S. E. Santa Clara

Fisher, F. G. Stanford University

Stanford Univ.

Bacon, H. M.

$\begin{array}{ll}\text { McMaster } & \text { Glover } \\ \text { Colorado Springs } & \begin{array}{l}\text { Powers } \\ \text { Sabin } \\ \text { Hansman }\end{array} \\ \begin{array}{l}\text { Sisam } \\ \quad \text { Denver }\end{array} & \begin{array}{c}\text { Bilgery Coll. } \\ \text { Fort Collins } \\ \text { Bures }\end{array} \\ \text { Gunder }\end{array}$

\section{CONNECTICUT}

Middletow
Wesleyan
Arnold, H.
Camp, B. H.
Foster, M. C.
Howland
Milford
Rosenbaum,
New Hav
Yale Un
Carlson, B.
Day, A. S.
Dilworth
Dunford, N.
Engstrom
Fullerton
Hall, M.
Hille
Jones, A. W.

\section{Kovarik}

Leech

Page

Putnam, A. L.

\section{Fittere}

\section{Golden}

Loretto

Cook, R. M.

Doyle, T. C.

Durand, W. F.

Herriot

Manning, W. A.

Polya

. C.

chaeffer

. C.

imoshenko

Uspensky
Longley

Miles, E. J.

Miller, D. S.

Ore

Peabody

Strange

Swope

Tracey

Uhler

Whittemore

Wilson, W. A.

\section{U. S. Coast Guard Acad. \\ Dimick}

\section{Storrs}

Sedgewick, R. W. Univ. of Connecticut

Cheney

Montgomery, J. C.

Nordling

Sedgewick, C. H. W.

Hall, N. A.

Loring

New London

Connecticut Coll. Bower, J. W. Turner, A. E.

Scherberg

Wood, H. A.

West Hartford

Barron

Walker, G. L.

Webber, G. C. $\begin{array}{ll}\text { Harter } & \text { Makarov } \\ \text { Jones, R. W. } & \text { Rees, C. J. }\end{array}$

\section{DISTRICT OF COLUMBIA}

Cromwell
Demers
Dribin
Duffin
Evans, J. O.
Gurney

Herschfeld

Holley

Judd

Karush

Kwerel

Moulton, F. R.
Norton

Sasuly

Siller

Singleton, H. E.

Sinkov

Smith, W. H. 


Supnick
Tuckerman
Van Orstrand
Vickery
American Univ.
Spencer, D. E.
Catholic Univ. of
America
Daly
Finan
Jordan, H. A.
Landry
Ramler
Rice, J. N.
Georgetozen Univ.
Sohon

George Washing-
ton Univ.
Humphreys, W. J.
Johnston
Mears
Taylor, J. H.
Weida
Wrench
Howard Univ.
Branson
Woodard
Trinity Coll.
Maloney, T. M.
U. S. Dept. of
Agric.
Girshick
Thompson, J. M.

\section{FLORIDA}

\section{Brooksville}

Blake, R. G.

Camp Murphy

Koch

Coral Gables

Meyer, $\mathrm{H}$.

De Land

Faulkner

Greville

Snow

Blau

Gainesville
Univ. of Florida
Dostal
George
Lang
Phipps
Quade
Simpson, T. M.

Specht

Wilson, W. H. Lakeland

Reinsch Pensacola

Varineau

Patterson, G. W. Justice

Scott, W. M.

S. Navy Dept.

Coddington

Coombs

Crow

Eberlein

Fry, W. J.

Gilbarg

Goldberg

Holly

Kitchens

Larrivee

Parke

Penney

Quinn

Wertheimer

Whitmore

Zwillinger

U. S. War Dept.

Dantzig, G. B.

\section{GEORGIA}

Athens
Univ. of Georgia
Barrow
Beckwith, W. S.
Callaway
Stephens, R. P.
Atlanta
Hanson, W. T.

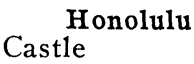

Caldwell

Rankin

Atlanta Univ.

Hefner

Pierce, J. A. Sewell

$$
\text { Clark Univ. Smith, D. M. }
$$

Dennis

Georgia School of Tech.

Carroll, C. L.

Steen

Webb

Decatur

Robinson, H. A.

\section{HAWAII}

Univ. of Hawaii Hoy, R. Y. Hoy, E. A.

\section{IDAHO}

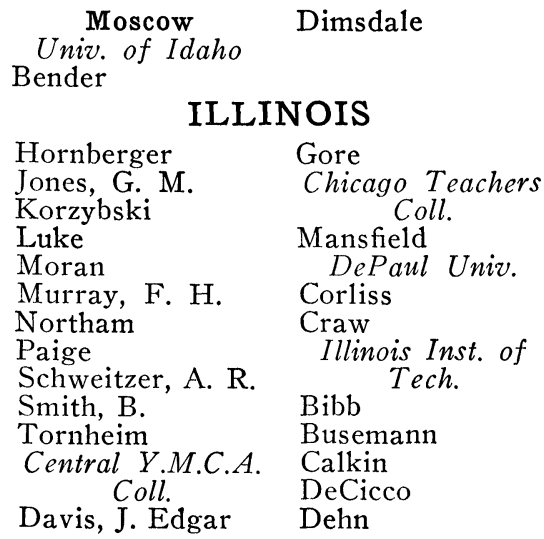

St. Petersburg

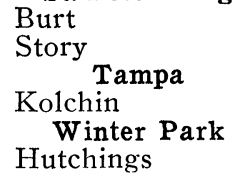

Fort Benning

Schetten

Milledgeville

Nelson, S. L. Rome

Hightower

Savannah

Williams, C. W.

Lihue

Hopwood

Taylor, E.

Ford, L. R.

Heins, M. H.

Krathwoh1

Mackey

Miser, H. J.

Oldenburger

Opatowski

Perlin

Sadowsky

Snyder, W. S.

Wilcox

Loyola Univ.

Gerst

Tordella 


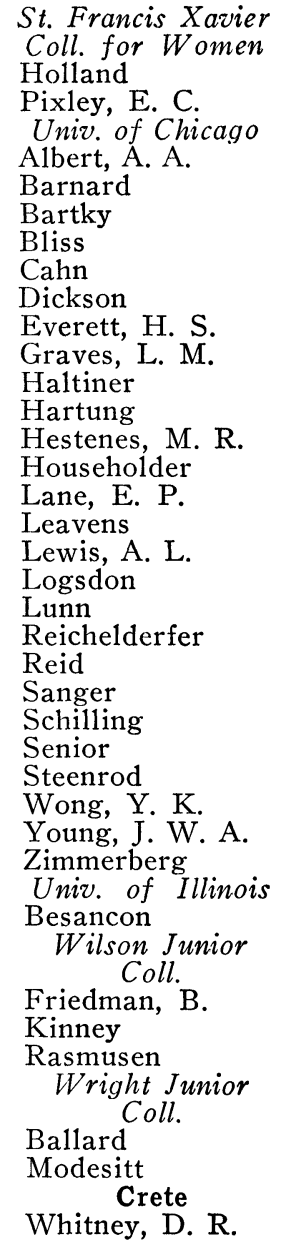

\section{Elsah \\ Hooper \\ Eureka}

Newson

Evanston

Carter, E. R.

Northwestern

Buell

$$
\text { Univ. }
$$

Curtiss, D. R.

Dobbie

Garabedian, H. L.

Givens

Griffiths

Hellinger

Holgate

Moulton, E. J.

Paydon

Peck, E. R.

Saastad

Scott, W. T.

Simmons, H. A

Stark, R. H.

Szatrowski

Wall

Wescott

Wetzel, M. D.

Wolfe, R. S.

Wood, F. E. Galesburg

Hurst, M. J. Knox Coll.

Sellew

Stephens, R. C. Joliet

Smith, F. C.

$$
\text { Kenilworth }
$$

Hart, W. W.

Curtis

Lake Forest

Fox, E.

Lisle
Shonka
Maywood
Baumgart
Olson, F. C. W.
Monmouth
Beveridge
Naperville
Keeler
Northbrook
Schweitzer, E. O.
Oak Park
Escott, E. B.
Lane, R. E.
Peoria
Comstock
Johanson
Rock Island
Cederberg
Rockton
Eddy
Scott Field
Garfin
McIaughlin
Springfield
Wells, N. W.
Urbana
Ketchum, G. S.
Univ. of Illinois
Alton
Armstrong
Atchison
Baer
Bailey, H. W.
Bell, J. C.
Bourgin
Bower, O. K.
Brahana
Bristow
Carmichael
Chanler
Coble
Crathorne

Day, M. M.

Doob

Emch

Ewing, F. E.

Fox, R. H.

Gibson, R. W.

Gingerich

Ginsberg

Halmos

Hamming

Hattan

Hazlett

Helton

Hoersch

Johnson, P. B.

Ketchum, P. W.

Kiefer

Kolsrud

Lapsley

Levy, $\mathrm{H}$.

Livesay

Lockhart

Mapes

Mathews

Mendel

Miles, H. J.

Miller, G. A.

Pepper, E. D.

Peters, J. W.

Price, J. F.

Schwartz, H. M.

Shanks, E. B.

Springer, M. D.

Staderman

Stewart, J. C.

Townsend

Trjitzinsky

Vaughan

Welker

Wilson, W.

Harris

\section{INDIANA}

\begin{tabular}{l}
\multicolumn{1}{c}{ Angola } \\
Moore, M. G. \\
Bloomington \\
Indiana Univ. \\
Artin \\
Hennel \\
Rothrock \\
Scherk \\
Stump \\
Weyl, F. J. \\
Williams, K. P. \\
Wolfe, H. E. \\
Crawfordsville \\
Polley \\
East Chicago \\
Burns, H. E.
\end{tabular}

$\begin{array}{cl}\text { Goshen } & \text { Arnold, B. H. } \\ \text { Hartzler } & \text { Ayres, W. L. } \\ \text { Greencastle } & \text { Burr } \\ \text { Edington } & \text { Clark, C. E. } \\ \text { Hanover } & \text { Fachus } \\ \text { Meyer, H. A. } & \text { Fine } \\ \text { Indianapolis } & \text { Giese } \\ \text { Cox, L. C. } & \text { Golomb } \\ \text { Creane } & \text { Graves, G. H. } \\ \text { Gingery } & \text { Hadley } \\ \text { Butler Univ. } & \text { Hazard, C. T. } \\ \text { Getchell } & \text { Heins, A. E. } \\ \text { Rodabaugh } & \text { Hodge } \\ \text { Lafayette } & \text { Hughes } \\ \text { Purdue Univ. } & \text { Lanczos } \\ \text { Alaoglu } & \text { Leibler }\end{array}$

Marshall

Niven

Olds, C. D.

Reade

Smith, A. H.

Stone, R. B.

Webster

Whitehead

Youngs

Notre Dame

Univ. of Notre Dame

Gassensmith

Kelley, J. L.

Kenna

Landin 


\begin{tabular}{lcc} 
Menger & \multicolumn{1}{c}{ Paoli } & Terre Haute \\
Milgram & Bentley & Palmer \\
Nash & St. Mary-of-the- & West Baden \\
Pepper, P. M. & Woods & Springs \\
Robinson, C. V. & Hayden & Lemmer
\end{tabular}

IOWA

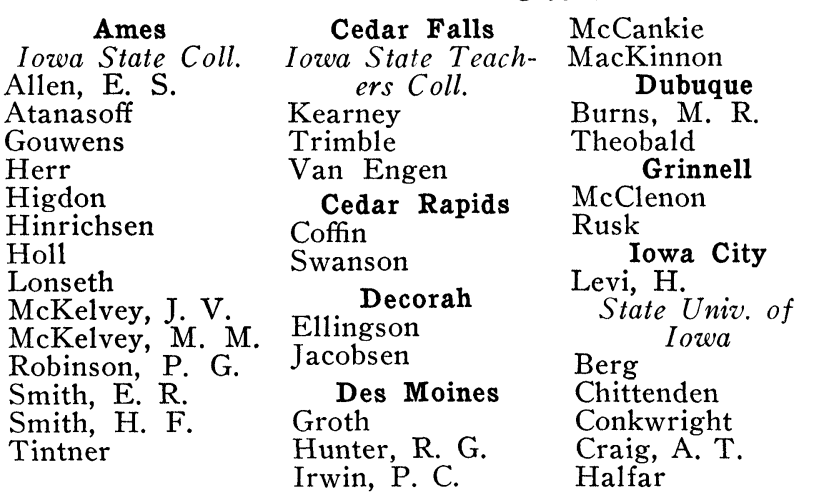

\section{KANSAS}

\begin{tabular}{|c|c|c|}
\hline Atchison & Black, F. L. & Daugherty \\
\hline Pretz & Jordan, H. E. & Janes \\
\hline Sullivan, $H$. & Kalisch & Mossman \\
\hline Emporia & Pate & Munro, G. C. \\
\hline Peterson, O. J. & Price, G. B. & Remick \\
\hline Tucker, C. B. & Stouffer & $\begin{array}{l}\text { Sigrey } \\
\text { Stratton }\end{array}$ \\
\hline $\begin{array}{l}\text { Independence } \\
\text { Bell, L. E. }\end{array}$ & $\begin{array}{l}\text { Wheeler, J. J. } \\
\text { Lindsborg }\end{array}$ & $\begin{array}{l}\text { North Newton } \\
\text { Richert }\end{array}$ \\
\hline $\begin{array}{c}\text { Lawrence } \\
\text { Univ. of Kansas }\end{array}$ & $\underset{\text { Manhattan }}{\text { Marm }}$ & $\begin{array}{l}\text { Pittsburg } \\
\text { Shirk }\end{array}$ \\
\hline $\begin{array}{l}\text { Babcock, W. } \\
\text { Bell, P. O. }\end{array}$ & $\begin{array}{l}\text { Kansas State Coll. } \\
\text { Babcock, R. W. }\end{array}$ & Smith, \\
\hline
\end{tabular}

\section{KENTUCKY}

Berea
Hutcherson
Bowling Green
Strayhorn
Yarbrough
Covington
Thuener
Lexington
Univ. of Kentucky
Boyd

Cohen, L. W.

Derthick

Downing

Howard

John

Latimer

LeStourgeon

Pence

Bullitt
Wolkow

Nazareth Coll.

Fowler, M. C.

Univ. of Louisville

Loewner

Moore, W. L.

Parker, S. T.

Stevenson

Carman
West Lafayette Westlund

Knowler

Oberg

Price, H. V.

Rietz

Ward, L. E.

Woods, R.

Mt. Vernon

Davis, W. M.

Moots

Rothe

Oskaloosa

Pella

Pietenpol

Sioux City

Graber

Rochford
Salina

Arnoldy

Jensen

Eberhart

Wichita

Longenecker

Caton
Richmond

Jenkins

Park, R. S.

Williamsburg

Boswell

\section{LOUISIANA}

Baton Rouge

Sanders, S. T.

Hammond

Cordrey

Lafayette

Loyola Univ.

Sanders, S. T., Jr. Larguier

Tulane Univ.

Cramer, G. F.

New Orleans

Buchanan, H. E.

Duren

Derby

Humphreys, M. G.

Many 


$\begin{array}{ll}\text { Thomson } & \text { Gentry } \\ \text { Weiss } & \text { Scotlandville } \\ \text { Xavier Univ. } & \text { Blackwell } \\ \text { Nightwine } & \text { Shreveport } \\ \text { Ruston } & \text { Cooper, C. M. } \\ \text { Gatewood } & \end{array}$

\author{
University \\ Louisiana State \\ Univ. \\ Anderson, P. H. \\ Carson \\ Dorroh \\ Harrold
}

\section{MAINE}

$\begin{array}{cl}\text { Brunswick } & \text { Holmes } \\ \text { Bowdoin Coll. } & \begin{array}{l}\text { Korgen } \\ \text { Moody }\end{array} \\ \text { Hhistie } & \text { Mommond }\end{array}$

Hammond

Aberdeen
Lorell
Aberdeen Proving
Ground
Dederick
Polachek
Annapolis
Muhly, I. F.
St. John's Coll.
Comenetz
U. S. Naval Acad.
Ayres, H. C.
Ball
Benac
Betz, E. E.
Bleick
Bramble
Church, R.
Clements
Cosby
Currier
Echols
Hawkins
Kells
Lindquist

Amherst

Amherst Coll. Brown, B. L.

Esty

Kleene

Sprague Belmont

Franklin, C. W. Boston

Coolidge, T. J.

Dysart

Garabedian, H. A.

Gould, A. B.

Miller, A. L.

Putnam, G.

Rines

Boston Univ.

Bruce

Mode
Korgen
Moody

\section{Orono \\ Univ. of Maine \\ Bryan \\ Comegys}

\section{MARYLAND}

McGaughey

Moore, T. W.

Muhly, H. T.

Phelps

Rawlins

Root

Scarborough

Seekins

Wagner, R. W.

Wilson, L. T.

\section{Gere

$$
\text { Arnold }
$$ \\ Baltimore}

Dawkins

Lukacs

Robinson, L. B.

Sutton, F. D. Goucher Coll.

Bacon, C. L.

Lewis, F. P.

Torrey

Bankie

Barten

Bourne

Bowie, C.

Bradshaw, C. H.

Cohen, A.

Curran

Dowker

Kershner

Larsen

Light

Morrill

Murnaghan

Naim

Peck, L. G.

Reed, L. J.

Seidenberg

Sherman, B.

Whaples

Wintner

Zariski Junior Coll.

Thomas, M. P.

Chestertown Univ.

\section{MASSACHUSETTS}

Harvard Univ.

Brown, T. H

Wilson, E. B. Northeastern Univ.

Haskins, E. E.

Spear

Suffolk Univ.

Sutton, F. X.

$$
\text { Cambridge }
$$

Sternberg

\section{Charnes}

Clifford, H. E.

Coolidge, J. L.

Hewitt

Huntington

Kaplansky

Kelley, T. L.

Kemble

Levit

Loomis

Lorenz

Lyman

MacLane, S.

Wilson, E. W.

Harvard Univ.

Beatley

Birkhoff, G.

von Mises

Norris, M. J.

Osgood

Phillips, R. S.

Pipes

Pollard, H.

Caywood
Mt. St. Agnes

Robinson, W. J.

Karnes

Nielsen

Parker, W. V.

Rickey

Robinson, V. N.

Rutt

Smith, H. L.

Kales

Kimball, S. H. Waterville

Galbraith

\section{College Park}

Univ. of Maryland

Dantzig, T.

Jackson, S. B.

Lancaster

Martin, M. H.

Newell

Vanderslice

Wagner, T. C. G.

Williams, M. H.

$$
\text { Cumberland }
$$

Rawhauser Frostburg

Hallett Hagerstown

Serbin

Harkin Hyattsville

Spicer Westminster

Spice Woodstock

McCauley
Rickart

Rulon

Shapley

Sheffer, H. M.

Stewart, F. M.

Stone, M. H.

Van Vleck, J. H.

Walsh

Westergaard

Whiteman

Whitney, $\mathrm{H}$.

Widder, D. V.

Massachusetts Inst. of Tech.

Arnold, K. J.

Barta

Cameron, R. H.

Clifford, A. H.

Crout 
Farley

Franklin, $\mathrm{P}$.

Harvey, G. G.

Heller

Hildebrand

LaSalle

Levinson, $\mathrm{N}$.

Loud

MacLane, G. R.

Martin, W. T.

Moon

Owens, G.

Phillips, H. B.

Pinney

Reissner

Salem

Samuelson

Struik, D. J.

Thomas, G. B.

Vallarta

Wallace, P. R.

Wallman

Wiener

Woods, F. S.

Yagi

Zeldin

$\quad$ Ann Arbor
Ellis, W.
Forsythe, G. E.
Grennan
Univ. of Michigan
Bartels
Beckenbach
Bradshaw, J. W.
Churchill
Civin
Coe
Copeland, A. H.
Craig, C. C.
Dushnik
Duthie
Dwyer
Eilenberg
Field
Fischer
Goldstine
Grau
Hay
Hildebrandt, T. H.
Hopkins, L. A.
Kaplan, S.

\section{Collegeville}

Kalinowski Duluth

Cothran

Kirchen

Hibbing

Erickson, R. W.
Radcliffe Coll.

Adams, R. B.

Graustein

Struik, S. R. R.

Chestnut Hill

Marcou

O'Donnell Chicopee

Madden Hyannis

McMullen Ipswich

Kinsman Lynn

Bauer Medford

Barnes Northampton Munroe, F. L. Smith Coll.

LeCaine

McCoy, N. H.

Montgomery, D.

Rambo
Norton

Garabedian, C. A. Pittsfield

Washburne

Sheffield

Sturley

Southbridge

Boeder

South Hadley

Mt. Holyoke Coll.

Litzinger

Nilson

Zygmund Springfield

Bowie, H. E. Swampscott

Evans, G. W. Tyngsboro

Richmond, C. A. Wellesley

Wellesley Coll.

Copeland, L. P.

Hazard, K. E.

Merrill, H. A.

Russell

Schuettler

\section{MICHIGAN}

Kaplan, W.

Karpinski

Kazarinoff

Love

Myers, S. B.

Nesbitt

Nyswander

Poor, V. C.

Rainich

Rainville

Running

Schilansky

Thorne, C. J.

Thrall

Wilder, R. L.

Bagby Detroit

Blankinship

Dunford, M. J.

Fleiger

Jones, C. D.

Levin, J. H.

Odle

Sugar

Tolonen

Weinberg

Great Lakes Coll.

Denton

Univ. of Detroit

Butler, J. F.

Hausmann

McCarthy, E. D.

Vezeau

Wayne Univ.

Borgman

Coral

Folley

Friedman, M.

Morrow, D. C.

Nelson, A. L.

Paxson

Pixley, H. H.

Southard

East Lansing

Michigan State

Beth

Blanche

Grove, V. G.

Heyda

\section{MINNESOTA}

$\quad$ Minneapolis
Bearman
Koehler, F.
Polansky
Thornton
Univ. of Minnesota
Amundson

Brink

Bussey

Campaigne

Carlson, E.

Crawford, W. S. H.

Dalaker

Gibbens
Smith, C. E.

Stark, M. E.

Young, M. M. Weston

Burke, L. Williamstown Williams Coll.

Agard

Hardy, J. G.

Richmond, D. E.

Shepard

Stoll

Wells, V. H.

Wray

Worcester

Wheeler, A. H.

Holy Cross Coll.

McCormick

O'Callahan

Worcester Poly-

Gay tech. Inst.

Morley

Rice, $\mathrm{H}$

Hill, J. D.

Hurd

Kibbey

Mitchell, W. L.

Plant

Powell

Sheedy

Speeker

Stewart, B. M.

Van Schaack

Wells, C. P.

Welmers

Zimmer

Fenton

Tryon

Highland Park

Schneider

Kalamazoo

Walton, T. O.

Marquette

Spooner

Owosso

Stewart, S. W. Ypsilanti

Schneckenburger

Hart, W. L.

Hill, E. L.

Jackson, D.

McEwen, W. R.

Montgomery, A. G.

Munro, W. D.

Ness 
Olmsted

Peebles

Scammon

Shumway

Spitzbart

Turrittin

Underhill

Blue Mountain

Trott

Cleveland

Ward, J. A.

$$
\text { Decatur }
$$

Dearman

\title{
Cape Girardeau
}

Michel

Rosskopf

$$
\text { Clayton }
$$

Haynes

Columbia

Univ. of Missouri

Betz, $\mathrm{H}$

Blumenthal

Ewing, G. M.

Ferguson, W. E.

Gillam

Schweigert

Shanks, M. E.

Thurman

Wahlin

Wehausen

Bozeman

Hurst, J. W.

Smith, C. B.

Lincoln
Arnold, H. A.
Union Coll.
Ogden
Univ. of Nebraska
Basoco

Brenke

Camp, C. C.

Candy

Collins

Doole

Gaba

Moorhead

Anderson, M. R. Northfield

Norlie

Rochester

Hickman

\author{
St. Paul \\ Coll. of St. Cath- \\ erine
Coll. of St. Thomas \\ Wegner \\ Bush, L. E.
}

\section{MISSISSIPPI}

$\begin{array}{cl}\text { Jackson } & \text { Walker, B. M. } \\ \text { McCoy, D. } & \text { Welch, F. P. } \\ \text { State College } & \text { University } \\ \text { Mississippi State } & \text { Hume } \\ \text { Coll. } & \text { Robinson, L. V. }\end{array}$

\section{MISSOURI}

Westfall

Burcham ayette

Basye

Melcher

Rockhurst Coll.

Doyle, W. C.

St. Teresa's Coll.

Lackay

$$
\text { Kirksville }
$$

Noble, C. E. Lexington

Miller, F. A.

Richmond Heights

Oberbeck

Roanoke

Ferguson, W. A. Rolla

Erkiletian

$$
\text { St. Louis }
$$

Cronvich

Green, F. W.

Rafferty

Short

Shreve

Maryville Coll.

Kernaghan

St. Louis Coll. of

DeFoe

$$
\text { Pharmacy }
$$

St. Louis Univ.

Case

\section{MONTANA}

$\begin{array}{cc}\text { Garrison } & \begin{array}{c}\text { Missoula } \\ \text { Montana State } \\ \text { Univ. }\end{array} \\ \text { Carey, E. F. A. A. }\end{array}$

\section{NEBRASKA}

C.

Neuhaus

Pierce, T. A.

Rock

Runge

Ear1

Omaha

NEVADA

Reno Vance

Univ. of Nevada Wood, F.

Beesley

\section{NEW HAMPSHIRE}

Concord
Conwell, G. M.
Durham
Univ. of New
Hampshire
Giddings

Lewis, D. C.

Slobin

Solt

Hanover
Dartmouth Coll. Brown, B. H. Forsyth

Haskins, C. N.

Mathewson
Landshoff

Thielman

Macalester Coll.

Camp, E. J.

French

Seiler

Winona

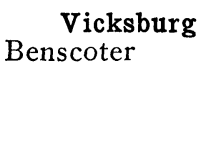

Regan

Ross

Washington Univ.

Avann

Dunkel

Rider

Roever

Warschawski Sedalia

Mayer, J. I. Springfield

H'Doubler

Finkel

Graves, W. L.

Warrensburg

Urban

Chatland

Dubisch

Lennes

Merrill, A. S.

Fitzpatrick

Hove

$$
\text { Wayne }
$$

Perkins, F. W.

Robinson, R.

Silverman

Wilder, C. E. 


Barber
Pratt
Belleplain
Durrell
Caldwell
Fowler, F. H.
Convent Station
O'Neill
Elizabeth
Nagle
Fort Monmouth
Rosenthal, J. E.
Hightstown
Litterick
Hoboken
Stevens Inst. of
Tech.
Davis, H. N.
Deimel
Hazeltine
Jacobus
Korn
Olmstead
Jersey City
McGrath
Solomon
Long Branch
Helly Madison
Knobelauch
Moorestown
Epstein, M. G.

Rosenthal, A.
Newsom

\section{NEW JERSEY}

$\begin{array}{lll}\begin{array}{c}\text { Mountain Lakes } \\ \text { Newark }\end{array} & \text { Cohen, I. S. } & \text { Segal } \\ \text { Hotelling, S. E. } & \text { Gubini } & \text { Singleton, R. R. } \\ \text { Bartleson } & \text { Maharam } & \text { Snapper } \\ \text { Brown, A. A. F. } & \text { Mayer, W. } & \text { Tompkins } \\ \text { Bulloch } & \text { Morse, M. } & \text { Tuan } \\ \text { Levinson, H. C. } & \text { von Neumann } & \text { Tucker, A. W. } \\ \text { Mosesson } & \text { Stone, A. H. } & \text { Tukey } \\ \text { Rhodes } & \text { Transue } & \text { Votaw } \\ \text { Thompson, J. S. } & \text { Veblen } & \text { Wedderburn } \\ \text { Widmark } & \text { Wade, L. I. } & \text { Wigner } \\ \text { Newark Coll. of } & \text { Weyl, H. } & \text { Wilks } \\ \text { Engineering } & \text { Wilkins } & \text { Rumson } \\ \text { Fithian } & \text { Princeton Univ. } & \text { Greenwald } \\ \text { New Brunswick } & \text { Adams, E. P. } & \text { South Orange } \\ \text { Rutgers Univ. } & \text { Ambrose } & \text { Rauch, L. M. } \\ \text { Brasefield } & \text { Anderson, T. W. } & \text { Stanwick } \\ \text { Bunyan } & \text { Bochner } & \text { Trenton } \\ \text { Grant, H. S. } & \text { Bohnenblust } & \text { Shuster } \\ \text { Kleinschmidt } & \text { Brown, G. W. } & \text { Upper Montclair } \\ \text { Meder } & \text { Chevalley } & \text { Cainpbell, G. A. } \\ \text { Morris, R. } & \text { Church, A. } & \text { New Jersey State } \\ \text { Nelson, C. A. } & \text { Eisenhart, L. P. } & \text { Teachers Coll. } \\ \text { Robertson, M. S. } & \text { Flood } & \text { Davis, D. R. } \\ \text { Starke } & \text { Gillespie } & \text { Hildebrandt, E. H. } \\ \text { Walter } & \text { Lefschetz } & \text { C. } \\ \text { Worth } & \text { McMillan } & \text { Mallory } \\ \text { Princeton } & \text { Montroll } & \text { West Orange } \\ \text { Inst. for Advanced } & \text { Rauch, L. L. } & \text { Edison } \\ \text { Study } & \text { Robertson, H. P. } & \text { Wannemacher } \\ \text { Alexander } & \text { Scheffé } & \\ \text { Bargmann } & & \\ & & \end{array}$

\section{NEW MEXICO}

$\begin{array}{lll}\text { Las Vegas } & \text { Roswell } & \text { State College } \\ \text { Rodgers } & \text { Alden } & \text { Joseph } \\ \text { Swingle }\end{array}$

\section{NEW YORK}

\section{Albany}

Frankel, E. T. Thompson, W. R. New York State Coll. for Teachers Beaver

Birchenough

DoBell

Lester

Stokes, E. C. Alfred

Lowenstein Aurora

Hollcroft, M. P. Wells Coll.

Carroll-Rusk

Clement, M. D.

Hollcroft, T. R. Beacon

Hammer, H.-K.

\section{Bethpage}

Tripp, R. H.

Brockport

Stopher

Bronxville

Gleason, A. M.

Brooklyn

Annenberg

Blake, E. M.

Hopkins, M. W.

Karlin

Kramer-Lassar

Locke

Miller, J. L.

Wynne

$$
\text { Brooklyn Coll. }
$$

Boeker

Borofsky

Boyer
Greenspan

Griffin, $\mathrm{H}$.

Johnson, R. A.

Kennison

Kormes, J. P.

Landers, A. W.

McMahon

MacNeish

Maria, A. J.

Maria, D. H.

Moore, L. T.

Prenowitz

Richardson, M.

Singer, J.

Wolfe, H.

Wolfe, J.

Woodbridge

Long Island Univ.

Lieber, H. G.

Lieber, L. R.
Polytech. Inst. of Brooklyn

Berry, W. J.

Bond

Fenn

Greeley

Reagen

Weber

Whitford

Pratt Inst.

Cowles

Thompson, J. E.

St. John's Univ.

Cassidy

Cowan

McCarthy, J. J.

Tolle

St. Joseph's Coll. for Women

Larkin 


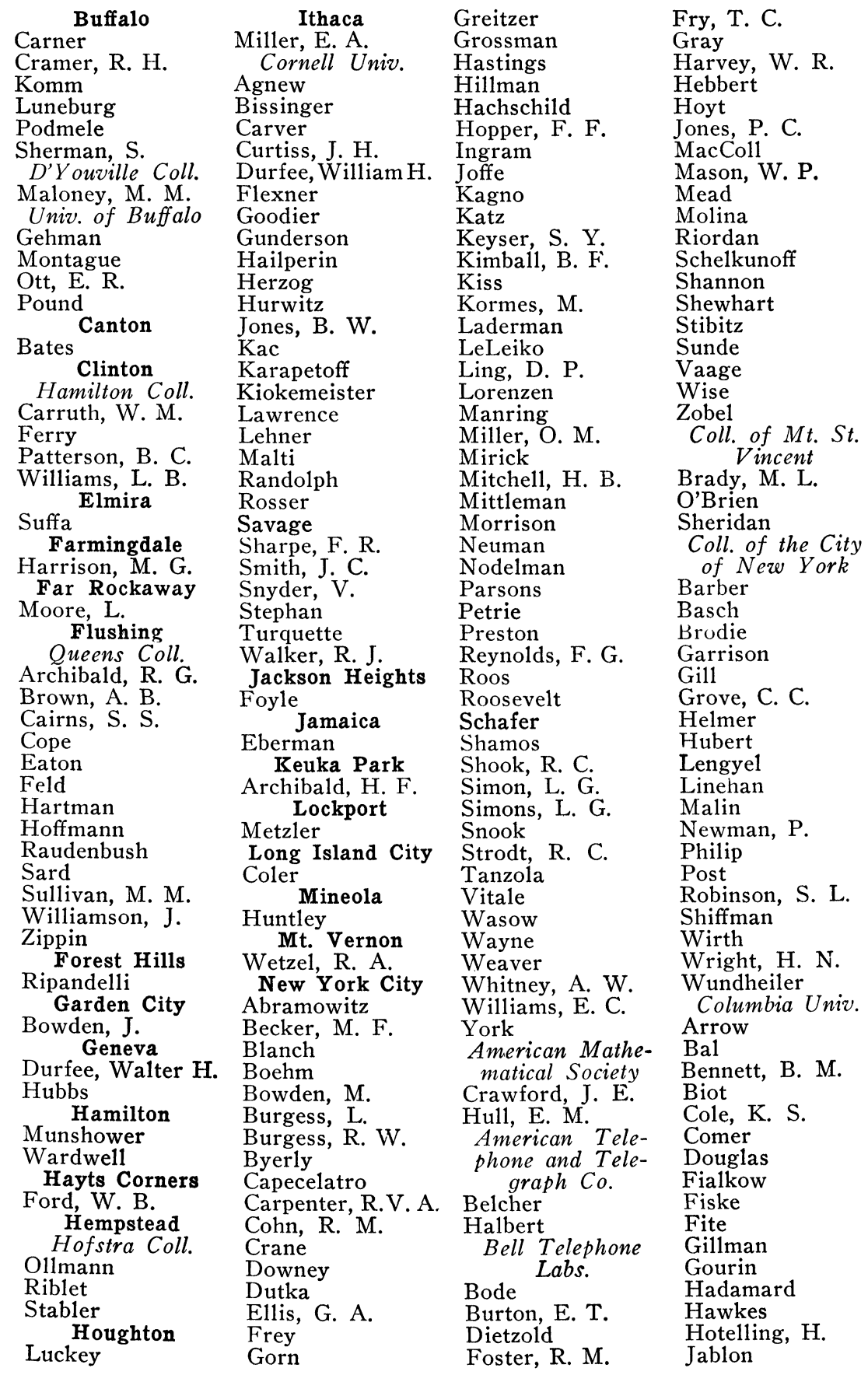




\begin{tabular}{|c|c|c|c|}
\hline & & & \\
\hline Kasner & kow & ers & ng, \\
\hline Keyser, C. J. & Elveback & Friedrichs & Seidel \\
\hline Koopman & Guggenbühl & $\mathrm{m}$ & Watkeys \\
\hline Ladue & Gutzman & M. & Rockville Cent \\
\hline Lorch, E. R. & Hall, F. C. & insey & Grant, A. M. C. \\
\hline Mann, H. B. & Hill, L. S. & $\mathrm{Ma}$ & Rocky Point \\
\hline Martin, M. P. & Landers, M. K. & e $\mathrm{s}$ & Carter, P. S. \\
\hline ins ay, F. J. & $\begin{array}{l}\text { A, M. } \\
\text { Duffee }\end{array}$ & $\begin{array}{l}\text { rs, A. S. } \\
\text { am, R. G }\end{array}$ & $\begin{array}{l}\text { St. Albans } \\
\text { Adler, C. F. }\end{array}$ \\
\hline$a_{y}, 1.0$ & Norris, N. & erg & St. Bonaventure \\
\hline $\cot t$ & M. & r, I. F. & Scheier \\
\hline ffer & er & Robbins, H. E. & Schenectady \\
\hline $\begin{array}{l}\text { Poor, C. L. } \\
\text { Ramsey }\end{array}$ & ner & Roth, S. G. & Alger \\
\hline ey & Whelan & & Poritsky \\
\hline Ritt & Manhattan Coll. & Tilley & Union Coll. \\
\hline Siceloff & Hett & Yanosik & Burkett \\
\hline Smith, D. E. & Welch, B. A. & Teachers Ins. and & Farrell \\
\hline h, P. A. & Manhattan Life & Annuity Asso. & $\begin{array}{l}\text { Fox, A. H. } \\
\text { Garis }\end{array}$ \\
\hline it, W, C. & $\begin{array}{l}\text { Ins. Co. } \\
\text { Porter, E. A. }\end{array}$ & $\begin{array}{l}\text { KobDrins, } \mathrm{K} . \text { B. } \\
\text { Yeshiva Coll. }\end{array}$ & $\begin{array}{l}\text { Garis } \\
\text { Morse, D. S. }\end{array}$ \\
\hline erland & Manhattanville & Gins & Snyder, A. D. \\
\hline & $\begin{array}{l}\text { Coll. of the } \\
\text { Sacred Heart }\end{array}$ & $\begin{array}{l}\text { Lowan } \\
\text { Matz }\end{array}$ & $\begin{array}{l}\text { Staten Isla } \\
\text { Wernick }\end{array}$ \\
\hline nberg & Kirby, M. D. & Nia & Syracuse \\
\hline $\begin{array}{l}\text { Williams, H. B. } \\
\text { Wolfowitz }\end{array}$ & Mira & Uni & Roe \\
\hline $\begin{array}{l}\text { Wolfowitz } \\
\text { Cooper Union }\end{array}$ & $\begin{array}{l}\text { Metropolitan Life } \\
\text { Ins. Co. }\end{array}$ & Banks & $\begin{array}{l}\text { Syracuse Univ. } \\
\text { Campbell, A. D. }\end{array}$ \\
\hline $\begin{array}{l}\text { Lehmann } \\
\text { MacDonald }\end{array}$ & Alfieri & Bush, K. & 11, I. S. \\
\hline $\begin{array}{l}\text { onald } \\
r, F . H .\end{array}$ & ford & Newton, A. V. & $\mathrm{Cc}$ \\
\hline $\begin{array}{l}\text { Miller, F. H. } \\
\text { Reddick }\end{array}$ & s, M. E. & Per & Decker \\
\hline $\begin{array}{l}\text { Reddick } \\
\text { Equitable Life As- }\end{array}$ & ilton, J. A. & Walker, G. W. & $\begin{array}{c}\text { Troy } \\
\end{array}$ \\
\hline $\begin{array}{l}\text { Equitable Life As- } \\
\text { surance Society of }\end{array}$ & $\mathrm{He}$ & $\begin{array}{l}\text { Poughkeepsie } \\
\text { Vassar Coll. }\end{array}$ & $\begin{array}{l}\text { Rensselaer Pols } \\
\text { tech Inst. }\end{array}$ \\
\hline $\begin{array}{c}\text { surance Society of } \\
\text { the U.S. }\end{array}$ & $\begin{array}{l}\text { Hohaus } \\
\text { Lotka }\end{array}$ & Baker, F. E. & Allen, E. B. \\
\hline $\begin{array}{l}\text { Murphy } \\
\text { Steinhaus }\end{array}$ & Mutual Life Ins. & Hopper, G. M. & Ames, D. B. \\
\hline $\begin{array}{l}\text { Steinhaus } \\
\text { Fordham Univ. }\end{array}$ & $\begin{array}{l}\text { Co. of N.Y. } \\
\text { Monsky }\end{array}$ & $\begin{array}{l}\text { McMillan, A. W. } \\
\text { Smith, G. }\end{array}$ & on, H. K. \\
\hline Kirby, A. R. & New School for & Wells, M. E. & Rempfer \\
\hline $\mathrm{K}$ & ial Rese & White, H. S. & West Point \\
\hline & Gun & Roch & rsoll \\
\hline augh & York Univ. & Betz, W. & Jones, H. \\
\hline & dourian & sna & Willseyville \\
\hline Hunter Coll. & & $\mathrm{F}$ & Seely \\
\hline $\begin{array}{l}\text { Anderson, R. L. } \\
\text { Aroian }\end{array}$ & stein, F. & zberg & W \\
\hline $\begin{array}{l}\mathrm{A} \\
\mathrm{B}\end{array}$ & $\stackrel{\mathrm{C}}{\mathrm{C}}$ & of $R$ & Ha \\
\hline , H. & & & \\
\hline$y$, J. H. & & G & \\
\hline $\mathrm{F}$ & zeiler & en, & \\
\hline & H & I & \\
\hline Camp Davis & ey & $\mathrm{Ja}$ & Gr \\
\hline Noether & Mackie & & ReBarker \\
\hline Chapel Hill & Durham & rve & Raleigh \\
\hline Univ. of North & Boas, R. P. & Roberts, J. H. & Efird \\
\hline $\begin{array}{l}\text { Carolina } \\
\text { uer, A. T. }\end{array}$ & , M. L. & North $\mathcal{C}$ & $\begin{array}{l}\text { North Carolin } \\
\text { State Coll. }\end{array}$ \\
\hline & itz & Coll. for Negro & Bullock \\
\hline Cameron, E & Dressel & Elder, A. & Cell \\
\hline bs & $\begin{array}{l}\text { Elliott, W. W. } \\
\text { Gergen }\end{array}$ & & $\begin{array}{l}\text { Clarkson, J. M. } \\
\text { Fisher, H. A. }\end{array}$ \\
\hline & & & \\
\hline
\end{tabular}


Kieval

Levine

Mumford
Nahikian
Park, H. V.
Strobel

\author{
Winton \\ Salisbury \\ Dearborn
}

\section{NORTH DAKOTA}

Fargo

Smith, I. W.

\section{OHIO}

Ada
Fulton Allance
Alian
Hildner
Athens
Ohio Uniz'.
Denbow
Marquis
Miller, D. D.
Reed, F. W.
Starcher
Bluffton
Linscheid
Bowling Green
Bowling Green
State Unio.
Hendrickson
Ogg
Overman
Bryan
Gardner
Cincinnati
Folz
Horst
Learson
Ryan
Our Lady of
Cincinnati Coll.
Reingold

Reingold

Univ. of Cincinnati

Barlaz

Barnett, I. A.

Brand

Farnau

Hancock

Horvay

Justice

Kersten

Lubin

Merriman

Miller, H. L.

Moore, C. $\perp$.

\section{Bartlesville}

Morris, W. L.

Forrest

Langston

LaFon

$$
\text { Norman }
$$

Univ. of Okla-

Brixey

\begin{tabular}{|c|c|}
\hline Rogers & Bareis \\
\hline Smith, E. S. & Blumberg \\
\hline Szász & Kuhn \\
\hline Taylor, W. C. & LaPaz \\
\hline White, E. F. & Rado \\
\hline Yowell & Rasor \\
\hline $\begin{array}{l}\text { Xavier Univ. } \\
\text { Stechschulte }\end{array}$ & Toops \\
\hline $\begin{array}{l}\text { Stechschulte } \\
\text { Cleveland }\end{array}$ & Wylie \\
\hline Brainard & Eagle \\
\hline Burwell & Kemmer \\
\hline Carruth, P. W. & Midgley \\
\hline Kraft & Schart \\
\hline $\begin{array}{l}\text { Case School of } \\
\text { Applied Science }\end{array}$ & $\begin{array}{l}\text { Univ. of Dayton } \\
\text { Schraut }\end{array}$ \\
\hline Brown, O. E. & Defiance \\
\hline Burington & MacCullough \\
\hline $\begin{array}{l}\text { Focke } \\
\text { Green, L. J. }\end{array}$ & Delaware \\
\hline $\begin{array}{l}\text { Green, L. J. } \\
\text { Guenther }\end{array}$ & Rowland \\
\hline McCuskey & Bumer \\
\hline $\begin{array}{l}\text { Morris, M. } \\
\text { Rinehart }\end{array}$ & MacNeille \\
\hline Sorgenfrey & Granville \\
\hline $\begin{array}{l}\text { Thomas, C. F. } \\
\text { Torrance, } \text { C. }\end{array}$ & $\begin{array}{l}\text { Denison Univ. } \\
\text { Kato }\end{array}$ \\
\hline $\begin{array}{l}\text { Torrance, C. C. } \\
\text { Fenn Coll. } \\
\text { Godfrey }\end{array}$ & $\begin{array}{l}\text { Kato } \\
\text { Ladner } \\
\text { Wiley }\end{array}$ \\
\hline $\begin{array}{l}\text { Patterson, W. A. } \\
\text { Tolar }\end{array}$ & Clarke \\
\hline $\begin{array}{l}\text { Topp } \\
\text { Western Reserve } \\
\quad \text { Univ. }\end{array}$ & $\begin{array}{l}\text { Kent } \\
\text { Kent State Univ. } \\
\text { Brooks }\end{array}$ \\
\hline Boyce & Harshbarger \\
\hline Musselman & Manchester \\
\hline $\begin{array}{l}\text { Simon, W. G. } \\
\text { Cleveland Heights }\end{array}$ & $\begin{array}{l}\text { Olson, E. J. } \\
\text { Stelson }\end{array}$ \\
\hline $\begin{array}{l}\text { Torrance, E. M. } \\
\text { Columbus }\end{array}$ & $\begin{array}{l}\text { Mariemont } \\
\text { Davids }\end{array}$ \\
\hline $\begin{array}{l}\text { Ohio State Unio. } \\
\text { Albert, G. E. }\end{array}$ & Leach \\
\hline
\end{tabular}

Bamforth

\section{OKLAHOMA}

Court

Dixon, W. J.

Hassler

McFarland

Meacham

Randels

Reaves

Springer, C. E.

\section{Stillwater}

Oklahoma Agric. and Mech. Coll.

Barnett, J.

Diamond

Hamilton, O. H.

Lewis, P. E.

Oboukhoff
Wilson

Smith, R. E.
Marietta

Bennett, $\mathrm{T}$.

Sandt

Mt. St. Joseph

Corona

North Baltimore

Blackall

$$
\text { Oberlin }
$$

Oberlin Coll.

Cairns, W. D.

Johnson, M. M.

Sinclair

Yeaton

Oxford

Miami Univ.

Anderson, W. E.

Pollard, H. S.

Western Coll.

Tappan

$$
\text { Painesville }
$$

Peters, R. M.

South Euclid

Garvin

$$
\text { Springfield }
$$

Tripp, M. O.

$$
\text { Toledo }
$$

Brandeberry

Ruger

Wellington

\section{Wilberforce}

Davidson

Wilmington

Spinks

Wooster

Coll. of Wooster

Meyer, H. L.

Williamson, C. O.

Yanney

Yellow Springs

Astrachan

Smith, H. W. Tulsa

Ellis, J. R.

Hodell

Jackson, O. B.

Ricker

Sharpe, J. A. 


Albany
Porter, R. E.
Corvallis
Oregon Stote Coll.
Hammer, P. C.
Hostetter
Manning, R.

Deck

Koehler, T. L. Annville

Black, A. H. Bethlehem

Smiley, D. M.

Beale

Bergmann

Cutler

Ewing, (W.) M.

Fort

Lamson

Latshaw

Pitcher, (A.) E.

Pond

Raynor

Shook, C. A.

Smail

Smiley, M. F.

Weil

\section{Bruceton}

Brown, F. W. Bryn Mawr

Reschovsky

Bryn Mawr Coll.

Geiringer

Lehr

Morrow, D. J.

Oxtoby

Wheeler, A. P. California

Bernstorf Carlisle

Ayres, F. Chambersburg Wilson Coll.

Davison

Disert

Johnson, R. F. Chester

Campbell, W. B.

Williams, F. G. Clarion

Carey, C. E. Dallas

Garrahan Drexel Hill

$\mathrm{McD}$ onough Dubois

Cunningham

\section{OREGON}

Milne, W. E. Kossack

Sobczyk

Maddaus

Moursund

$$
\text { Eugene }
$$

Univ. of Oregon

Bubb

DeCou

Portland

Ghent Reed Coll.

Griffin, F. L.

\section{PENNSYLVANIA}

Easton
Bailey, R. P.
Smith, W. M.
East Pittsburgh
Slepian
Gettysburg
Arms
Livingood
Glenside
Patterson, J. O.
Greensburg

Epstein, B.

Fernbach

Fudge

Groat

Hayward

Hudson

Jaffe

Kratz

McNeil Harrisburg

McKee

Whited

$$
\text { Haverford }
$$

Haverford Coll.

Allendoerfer

Gummere

Oakley

Wilson, A. H.

$$
\text { Hazleton }
$$

Herpel Immaculata

Anastasia Maria Jenkintown

Durand, J. C. Kutztown

Knedler Lancaster

Murray, W. R.

Yahner Latrobe

\section{Lewisburg}

Bucknell Univ.

\section{Drum}

Gold

Miller, W. I.

Lincoln University

Haviland

$$
\text { Meadville }
$$

Frame Narberth

Robertson, W. M. New Kensington

Sturm

New Wilmington

Black, H. L. Philadelphia

Beamish

Brown, R. D.

Constable
Morgan, W. B.

Smith, T. L.

Sherman, J.

Smith, J. P.

Drexel Inst. of Tech.

Davis, J. Elmer Temple Univ.

Gleason, R. E.

Lawton

Rorer

Stokes, C. N. Univ. of Penn-

Beal sylvania

Burks

Caris

Clarkson, J. A.

Dressler

Erdös

Evans, H. B.

Goff

Hemmingsen

Kline, J. R.

Lufkin

Mitchell, H. H.

Rademacher

Ritter, E. K.

Safford

Schoenberg

Shohat

Shugert

Wallace, A. D.

Whitman, P. M.

Witmer

Briant Pittsburgh

Leifer

Carnegie Inst. of Tech.

Clippinger

Dines

Hestenes, A. D.

Hicks for Women

Calkins

Boggs

Culver

Foraker

Knipp

Speer

Taylor, J. S.

Huff

$$
\text { Pottstown }
$$

Scranton

Trapani

Walton, M. B. Selinsgrove

Smith, A. J.

Owens, H. B.

Bentz State Coll.

Cohen, T.

Curry

Dotterer

Frink, A. H.

Frink, $\mathrm{O}$.

Gordon

Graves, C. H.

Hagen

Johnson, E.

Krall

Owens, F. W.

Rupp

Schwartz, A.

Sheffer, I. M.

Stecker

Umberger

Wagner, C. C.
Rosenbaum, L. J.

Rosenbaum, R. A.

Univ. of Portland

Vassallo

Luther

Olshen

Landau

Morehead

Moskovitz

Nelley

Olds, E. G.

Peach

Rosenbach

Saibel

Pennsylvania Coll.

Univ. of PittsState College Pennsylvania

Harrington, W. J. 


$\quad$ Swarthmor
Swann
Swarthmore
Brinkmann
Dresden


Baguio
Melchor
Manila
Jaramillo

Pease

Stauffer

Providence

Brown Univ.

Adams, C. R.

Archibald, R. C

Bennett, A. A.

Bergman

Carlen

Shulits
Coll. of Charles-
ton

Bushkovitch

Brookings
South Dakota
State Coll.
MacDougal

Pulliam Jackson
Rnoxville

$\quad$ Abilene
Burnam
Mullings
Angleton
Bright
Austin
Univ. of Texas
Batchelder
Coburn

Fried

Marriott

Miller, J. A.

Dorwart
Shaub

PHILIPPINE ISLANDS

Netzorg
Mapua Inst. of
Tech.

Mijares

\section{PUERTO RICO}

Mayaquez

Morales

Sánchez-Díaz

Rio Piedras

Bobonis

Kuschke

\section{RHODE ISLAND}

Feller

Gaskell

Gilman

Greenberg

Hall, D. W.

Hutchinson, L. C.

Lindsay

Manning, H. P.

Mintzer

Morkovin

Munroe, M. E.

Neugebauer

Prager

Protter

Providence Coll.

Sohl

Tamarkin

Western, D. W.

Richardson, R. G. McKenney D.

Riess

Ruley

Schmidt

Smiley, C. H.

Brant

West Warwick

SOUTH CAROLINA

Dye

The Citadel

Hedge

Sutton, C. S. Clemson

Coker

Columbia

Coleman, J. B.

Jackson, J. B.

Rock Hill

Royall

Stokes, R. W.

SOUTH DAKOTA

Walder

Wente

Rapid City

Sioux Falls

Cook, R. H.

Lintvedt

\section{TENNESSEE}

Univ. of Tenness
Eaves
Ficken
Memphis
MacQueen

Craig, H. V.
Decherd
Dodd
Ettlinger
Greenwood
Jones, F. B.
Lubben
Moore, R. L.
Porter, G. H.

Feather

Willow Grove

Bateman, P. T.

Perez

Virata 


Commerce
Wright, C. B.
Corpus Christi
Leavitt
Dallas
McDermott
Montgomery, P. V.
Southern Metho-
dist Univ.
Palmquist
Rees, P. K.
Starr
Denton
North Texas State
Teachers Coll.
Brown, M. C.
Hanson, E. H.
Miller, H. C.
Georgetown
Olson, H. L.

son, H. I

Logan
Bird Salt Lake City
Woodbury

Bennington
Polanyi
Burlington
Univ. of Vermont
Bullard

Arlington
Brenner
Newman, J. R.
Blacksburg
Virginia Polytech.
Inst.
Hatcher
McFadden
Williams, J. E.
Charlottesville
Univ. of Virginia
Bernard
Botts
Clark, C. L.
Cox, M. J.
Garcia, M.
Gottschalk
Hedlund

$\quad$ Houston
Rust
Slotnick
$\quad$ Rice Inst.
Bray
Brunk
Dean, A. C.
Leighton
Lovett
Mandelbrojt
Piranian
Thron
Ulrich
Wilson, H. A.
Univ. of Houston
Hutchinson, J. D.
$\quad$ Huntsville
Querry
Jacksonville
Clarkson, H. E.

Kelly Field

Trachtenberg

Kingsville

Kennedy, E. C.

Lubbock

Texas Tech. Coll.

Gilbert

Rigby

Thompson, E. I

Falconer Mercedes

Shaw, W. F.

Randolph Field

Seebeck

San Antonio

Incarnate Word

Gough Coll.

\section{UTAH}

$\begin{array}{ll}\text { Univ. of Utah } & \begin{array}{l}\text { Hayes, J. J. } \\ \text { Horsfall } \\ \text { Biesele }\end{array} \\ \text { Gibson, J. L. } & \text { Pehrson }\end{array}$

\section{VERMONT}

\author{
McClay \\ Swift, E. \\ Middlebury \\ Ballou
}

Terry

Springfield

\section{VIRGINIA}

Linfield

McShane

Myers, F. G.

Oglesby

Whyburn, G. T. Dahlgren

Barker Ettrick

Hunter, L. S. McDaniel, R. R.

Fredericksburg Carter, H. C.

Hampden-Sydney

Elliott, E. R.

$$
\text { Hampton }
$$

Lorch, L.

Street

Hampton Inst.

Perkins, H. A.

Simond

Hollins College

Allen, H. W.

Mitchell, J. M.

Fazel Hopewell

\section{Lexington}

Virginia Military Inst.

Byrne

Knox

Washington and Lee Univ.

Paxton

Larew

$$
\text { Lynchburg }
$$

\section{WASHINGTON}

Raymond

mond Pullman

Butler, L. G.

Knebelman

\section{Seattle}

Seattle Pacific Coll.

Beegle
Univ. of Washington

Ballantine, C. R

Ballantine, J. P.

Beaumont
Our Lady of the Lake Coll.

Hill, M. L.

St. Mary's Univ.

Becker, F. A.

San Antonio

Junior Coll.

Hurry

Tulloch

Trinity Univ.

Newton, G. A.

Sandidge Terrell

Hedberg

Williams, W. J.

Smith, S. S.

Stafford

Winooski Park

Gleyza1

Wiggin

South Boston

Patten

Staunton

Taylor, M. E.

\section{Sweet Briar}

Cole, N.

Morenus

University of

Richmond

Univ. of Richmond

Gaines

Grable

Wheeler, C. H.

Williamsburg

Phalen

Stetson

Birnbaum

Carpenter, A. F.

Copenhagen

Cramlet

Jerbert, A. H. 
Jerbert, A. R.

Kingston, J. M.

McFarlan

Mullemeister

Neikirk

Schaefer

Taub

Winger
Zuckerman Spokane

Martin, F. L.

Walla Walla

Bratton

Highberg

Woodinville

Anthony

\title{
WEST VIRGINIA
}

\author{
Cramblet \\ Bluefield \\ Porter, J. W.
}

Bethany

Morgantown

Hackney West Virginia Univ.

Davis, H. A.

Eiesland
Reynolds, C. N. Turner, B. M. Vest

Shepherdstown

Callahan

\section{WISCONSIN}

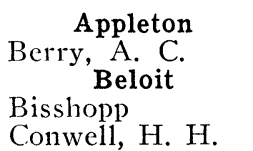

May

Finch

Univ. of Wisconsin

Adler, F. T.

Allen, $\mathrm{F}$. E.

Breit

Bruck

Colvin

Eisenhart, C.

Embree

Evans, H. P.

Everett, C. J.
VanVleck, E. B.

Vinograde

Wiegmann Milwaukee

Johnson, R. E.

Laden

Langer

Lee

Lindstrum

March

Nelson, N. D.

Norman

Rodd

Simpson, R. C.

Slichter

Smith, W. N.

Sokolnikoff, E. S. Norris, R. E.

Sokolnikoff, I. S. Univ. of Wisconsin

Swain

Trump

Ulam
Marquette Univ.

Pettit

Wilczewski MilwaukeeDowner Coll.

Beckwith, E. R. Mt. Mary Coll.

Vaudreuil

State Teachers Coll.

at Milwaukee

Bardell

Battig
Kenncy

Marden

Nordhaus

Roth, W. E.

Vass

Wolf, L. A.

Oshkosh

Beenken

Price, I. River Falls

Eide

Superior

Danielson

Smith, C. W.

West De Pere

DeCleene

Williams Bay

Chandrasekhar

\section{WYOMING}

$\begin{array}{ll}\text { Lamont } & \begin{array}{c}\text { Laramie } \\ \text { Bellamy }\end{array} \\ \text { Rechard } \\ \text { Samelson }\end{array}$




ALBERTA
Banff
Crosby
Edmonton
Univ. of Alberta
Campbell, J. W.
Cook, A. J.
Sheldon
BRITISH
COLUMBIA
Vancouver
Univ. of British
Columbia
Buchanan, D.
Gage
Hull, R.
Jennings, S. A.
MANITOBA
Winnipeg
Wilson, N. R.
NEW
BRUNSWICK
Fredericton
Miller, C. E.
Sackville
McEwen, W. H.

\section{CANADA}

NOVA SCOTIA
Halifax
Adshead
Walmsley
Stellarton
Loomer
Wolfville
Jeffery
Macphail
ONTARIO
Hamilton
Findlay
Johns
Kingston
Halperin
Miller, N.
London
Cole, R. H.
Kingston, H. R.
Orillia
Guinand
Ottawa
Bell, J. H.
Brady, G. V.
Fraser, W. C. G.
Mendelsohn

Toronto

Wolfenden

Ontario Coll. of Education

Grant, A. A.

O'Connor

Montreal

Loyola Coll.

Univ. of Toronto Pall

Beatty Shaw, A. N.

Brauer, R.

Burk

Coleman, A. J.

Coxeter

DeLury

Gilchrist

Gould, S. H.

Krieger

Pounder

Robinson, G. de B.

Synge

Waddell

Webber, W. J.

Weinstein QUEBEC

Lennoxville

Richardson, A. V.

Sullivan, C. T.

Williams, W. L. G.

Tremblaye

$$
\text { Westmount }
$$

Chapman

\section{SASKAT - \\ CHEW AN \\ Saskatoon}

Univ. of Saskatchezran

Derry

James, R. D.

Ling, G. $H$.

Murdoch

\title{
AFRICA
}

\section{SOUTH AFRICA}

de Wet

\author{
Pretoria
}

ASIA

$\begin{gathered}\text { CHINA } \\ \text { Peking }\end{gathered}$
Hsï Shanghai
Fan INDIA
IN Aligarh
Shah
Annamalainagar
Rao Bangalore
Iyengar
Dacca
Vijayaraghavan

Delhi
Seth
Madras
Ananda-Rau
Poona
Paranjpye
IRAQ
Baghdad
Merrick
JAPAN
Osaka
Kakutani
Nakayama
Sendai
Kubota
Takasu Tokyo
Kuniyeda
Yoshida
PALESTINE Jerusalem
Levitski

$$
\text { Nablus }
$$
Tukan
Petah Tikvah
Preiser

\author{
STRAITS \\ SETTLEMENTS \\ Singapore \\ Oppenheim \\ TURKEY \\ Istanbul \\ Kimball, W. S.
}

\section{AUSTRALASIA}

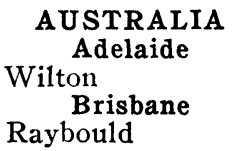
Perth
Weatherburn Sydney
Carslaw
Thorne, H. H.


EUROPE

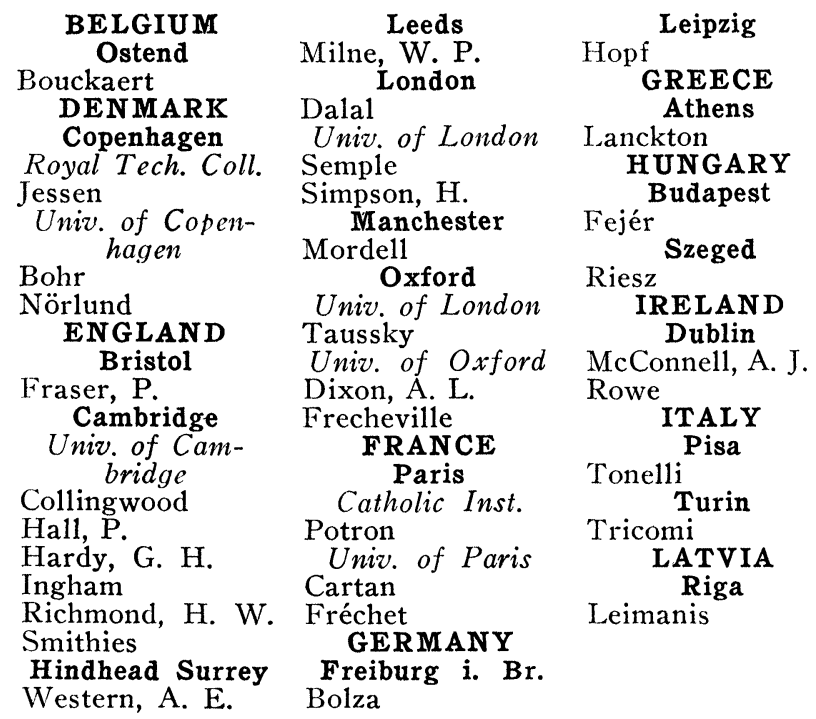
NORTHERN
IRELAND
Belfast

Queen

McCrea

Povey

SCOTLAND

Dundee

Copson

Edinburgh

Whittaker

SWEDEN

Stockholm

Oseen

SWITZERLAND

Lausanne

Blanc

Zurich

Plancherel U.S.S.R.

Kryloff

$$
\text { Ufa }
$$

WALES

Bangor

Berwick

Cardiff

Livens

\section{S}

CENTRAL AND SOUTH AMERICA

ARGENTINA
Buenos Aires
Univ. of Buenos
Aires
Baidaff
Barral-Souto
Blaquier
Bula
González-

Domínguez
La Plata

de Cesare

Rosario

Gaspar

Levi, B.

Tucumán

Terracini

CUBA

Havana

Corral-Aléman
González-

Rodríguez

ECUADOR

Thullen Quito

MEXICO

Mexico

Baños

PERU

Lima

Catholic Univ. of Peru de Losada y Puga Univ. of San Marcos

García, G.

Rosenblatt 